\title{
CHEBYSHEV'S BIAS FOR PRODUCTS OF TWO PRIMES
}

\author{
KEVIN FORD AND JASON SNEED
}

\begin{abstract}
Under two assumptions, we determine the distribution of the difference between two functions each counting the numbers $\leqslant x$ that are in a given arithmetic progression modulo $q$ and the product of two primes. The two assumptions are (i) the Extended Riemann Hypothesis for Dirichlet $L$-functions modulo $q$, and (ii) that the imaginary parts of the nontrivial zeros of these $L$-functions are linearly independent over the rationals. Our results are analogs of similar results proved for primes in arithmetic progressions by Rubinstein and Sarnak.
\end{abstract}

\section{INTRODUCTION}

1.1. Prime number races. Let $\pi(x ; q, a)$ denote the number of primes in the progression $a$ mod $q$. For fixed $q$, the functions $\pi(x ; q, a)$ (for $a \in A_{q}$, the set of residues coprime to $q$ ) all satisfy

$$
\pi(x, q, a) \sim \frac{x}{\varphi(q) \log x}
$$

where $\varphi$ is Euler's totient function [Da]. There are, however, curious inequities. For example $\pi(x ; 4,3) \geqslant$ $\pi(x ; 4,1)$ seems to hold for most $x$, an observation of Chebyshev from 1853 [Ch]. In fact, $\pi(x ; 4,3)<$ $\pi(x ; 4,1)$ for the first time at $x=26,861$ [Le]. More generally, one can ask various questions about the behavior of

$$
\Delta(x ; q, a, b):=\pi(x ; q, a)-\pi(x ; q, b)
$$

for distinct $a, b \in A_{q}$. Does $\Delta(x ; q, a, b)$ change sign infinitely often? Where is the first sign change? How many sign changes with $x \leqslant X$ ? What are the extreme values of $\Delta(x ; q, a, b)$ ? Such questions are colloquially known as prime race problems, and were studied extensively by Knapowski and Turán in a series of papers beginning with $[\mathrm{KT}]$. See the survey articles $[\mathrm{FK}]$ and $[\overline{\mathrm{GM}}]$ and references therein for an introduction to the subject and summary of major findings. Properties of Dirichlet $L$-functions lie at the heart of such investigations.

Despite the tendency for the function $\Delta(x ; 4,3,1)$ to be negative, Littlewood [Li] showed that it changes sign infinitely often. Similar results have been proved for other $q, a, b$ (see $[\mathbf{S}]$ and references therein). Still, in light of Chebyshev's observation, we can ask how frequently $\Delta(x ; q, a, b)$ is positive and how often it is negative. These questions are best addressed in the context of logarithmic density. A set $S$ of positive integers has logarithmic density

$$
\delta(S)=\lim _{x \rightarrow \infty} \frac{1}{\log x} \sum_{\substack{n \leqslant x \\ n \in S}} \frac{1}{n}
$$

provided the limit exists. Let $\delta(q, a, b)=\delta(P(q, a, b))$, where $P(q, a, b)$ is the set of integers $n$ with $\Delta(n ; q, a, b)>0$. In 1994, Rubinstein and Sarnak [RS] showed that $\delta(q ; a, b)$ exists, assuming two hypotheses (i) the Extended Riemann Hypothesis for Dirichlet $L$-functions modulo $q\left(\mathrm{ERH}_{q}\right)$, and (ii) the imaginary parts of zeros of each Dirichlet $L$-function are linearly independent over the rationals $\left(\mathrm{GSH}_{q}\right.$ Grand Simplicity Hypothesis). The authors also gave methods to accurately estimate the "bias", for example showing that $\delta(4 ; 3,1) \approx 0.996$ in Chebyshev's case. More generally, $\delta(q ; a, b)=\frac{1}{2}$ when $a$ and $b$ are either

Date: October 30, 2018.

2000 Mathematics Subject Classification:11M06, 11N13, 11 N25.

The research of K. F. was supported in part by National Science Foundation grants DMS-0555367 and DMS-0901339. 
both quadratic residues modulo $q$ or both quadratic nonresidues (unbiased prime races), but $\delta(q ; a, b)>\frac{1}{2}$ whenever $a$ is a quadratic non-residue and $b$ is a quadratic residue. A bit later we will discuss the reasons behind these phenomena. Sharp asymptotics for $\delta(q ; a, b)$ have recently been given by Fiorilli and Martin $[\overline{F M}]$, which explain other properties of these densities.

1.2. Quasi-prime races. In this paper we develop a parallel theory for comparison of functions $\pi_{2}(x ; q, a)$, the number of integers $\leqslant x$ which are in the progression $a \bmod q$ and which are the product of two primes $p_{1} p_{2}\left(p_{1}=p_{2}\right.$ allowed). Put

$$
\Delta_{2}(x ; q, a, b):=\pi_{2}(x ; q, a)-\pi_{2}(x ; q, b),
$$

let $P_{2}(q, a, b)$ be the set of integers $n$ with $\Delta_{2}(n ; q, a, b)>0$, and set $\delta_{2}(q, a, b)=\delta\left(P_{2}(q, a, b)\right)$. The table below shows all such quasi-primes up to 100 grouped in residue classes modulo 4 .

\begin{tabular}{|c|c|}
\hline$p q \equiv 1(\bmod 4)$ & $p q \equiv 3(\bmod 4)$ \\
\hline 9 & 15 \\
\hline 21 & 35 \\
\hline 25 & 39 \\
\hline 33 & 51 \\
\hline 49 & 55 \\
\hline 57 & 87 \\
\hline 65 & 91 \\
\hline 69 & 95 \\
\hline 77 & \\
\hline 85 & \\
\hline 93 & \\
\hline
\end{tabular}

Observe that $\Delta_{2}(x ; 4,3,1) \leqslant 0$ for $x \leqslant 100$, and in fact the smallest $x$ with $\Delta_{2}(x ; 4,3,1)>0$ is $x=26747$ (amazingly close to the first sign change of $\Delta(x ; 4,3,1)$ ). Some years ago Richard Hudson conjectured that the bias for products of two primes is always reversed from that of primes; i.e., $\delta_{2}(q ; a, b)<$ $\frac{1}{2}$ when $a$ is a quadratic non-residue modulo $q$ and $b$ is a quadratic residue. Under the same assumptions as [RS], namely $\mathrm{ERH}_{q}$ and $\mathrm{GSH}_{q}$, we confirm Hudson's conjecture and also show that the bias is less pronounced.

Theorem 1. Let $a, b$ be distinct elements of $A_{q}$. Assuming $\mathrm{ERH}_{q}$ and $\mathrm{GSH}_{q}, \delta_{2}(q ; a, b)$ exists. Moreover, if a and $b$ are both quadratic residues modulo $q$ or both quadratic non-residues, then $\delta_{2}(q ; a, b)=\frac{1}{2}$. Otherwise, if a is a quadratic nonresidue and $b$ is a quadratic residue, then

$$
1-\delta(q ; a, b)<\delta_{2}(q ; a, b)<\frac{1}{2} .
$$

We can accurately estimate $\delta_{2}(q ; a, b)$ borrowing methods from [RS, §4]. In particular we have

$$
\delta_{2}(4 ; 3,1) \approx 0.10572 .
$$

We deduce Theorem 1 by connecting the distribution of $\Delta_{2}(x ; q, a, b)$ with the distribution of $\Delta(x ; q, a, b)$. Although the relationship is "simple", there is no elementary way to derive it, say by writing

$$
\pi_{2}(x ; q, a)=\frac{1}{2} \sum_{p \leqslant x} \pi\left(\frac{x}{p} ; q, a p^{-1} \bmod q\right)+\frac{1}{2} \sum_{\substack{p \leqslant \sqrt{x} \\ p^{2} \equiv a(\bmod q)}} 1 .
$$

In particular, our result depends strongly on the assumption that the zeros of the $L$-functions modulo $q$ have only simple zeros. Let $N(q, a)$ be the number of $x \in A_{q}$ with $x^{2} \equiv a(\bmod q)$, and let $C(q)$ be the set of nonprincipal Dirichlet characters modulo $q$. 


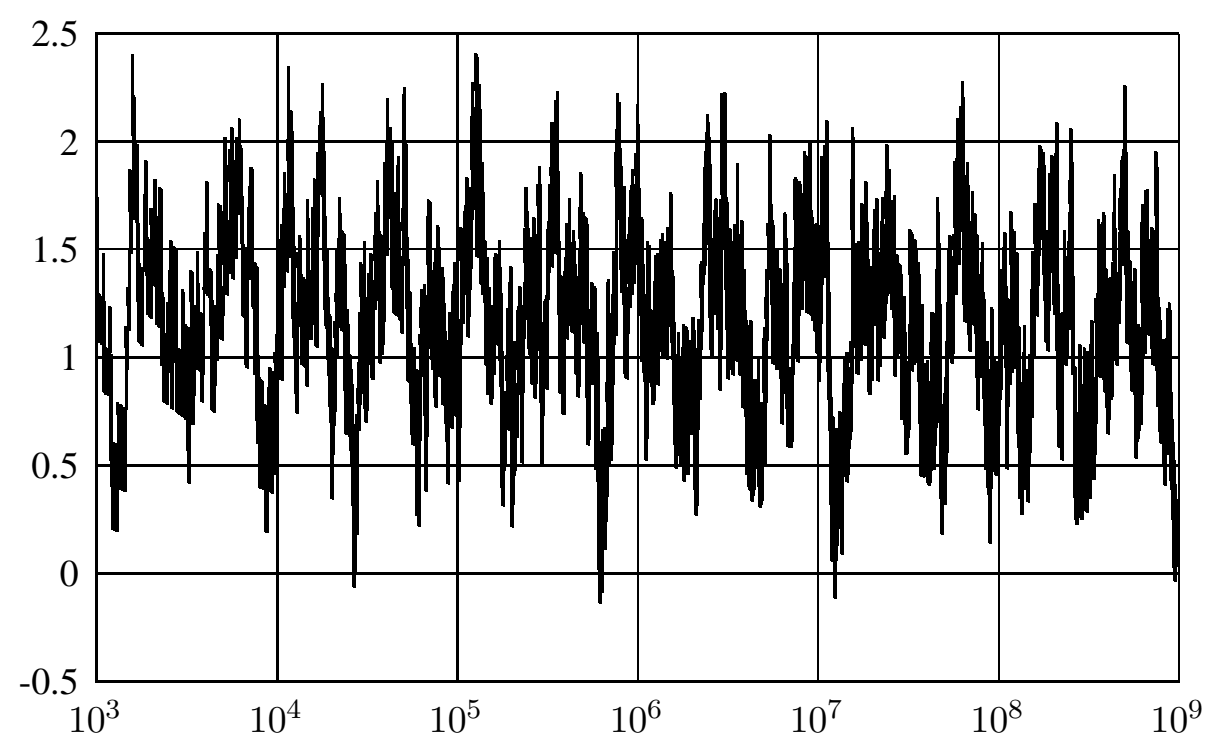

FIGURE 1. $\frac{\log x}{\sqrt{x}} \Delta(x ; 4,3,1)$

Theorem 2. Assume $\mathrm{ERH}_{q}$ and for each $\chi \in C(q), L\left(\frac{1}{2}, \chi\right) \neq 0$ and the zeros of $L(s, \chi)$ are simple. Then

$$
\frac{\Delta_{2}(x ; q, a, b) \log x}{\sqrt{x} \log \log x}=\frac{N(q, b)-N(q, a)}{2 \phi(q)}-\frac{\log x}{\sqrt{x}} \Delta(x ; q, a, b)+\Sigma(x ; q, a, b),
$$

where $\frac{1}{Y} \int_{1}^{Y}\left|\Sigma\left(e^{y} ; q, a, b\right)\right|^{2} d y=o(1)$ as $Y \rightarrow \infty$.

The expression for $\Delta_{2}$ given in Theorem 2 must be modified if some $L(s, \chi)$ has multiple zeros; see $\S 3$ for details.

Figures 1,2 and 3 show graphs corresponding to $(q, a, b)=(4,3,1)$, plotted on a logarithmic scale from $x=10^{3}$ to $x=10^{9}$. While $\Sigma(x ; 4,3,1)$ appears to be oscillating around -0.2 , this is caused by some terms in $\Sigma(x ; 4,3,1)$ of order $1 / \log \log x$, and $\log \log 10^{9} \approx 3.03$. By Theorem $2, \Sigma(x ; 4,3,1)$ will (assuming $\mathrm{ERH}_{4}$ and $\mathrm{GSH}_{4}$ ) eventually settle down to oscillating about 0 .

It is not immediate that Theorem 1 follows from Theorem 2 One first needs more precise information about the distribution of $\Delta(x ; q, a, b)$ from [RS].

Theorem RS. [RS, §1] Assume $\mathrm{ERH}_{q}$ and $\mathrm{GSH}_{q}$. For any distinct $a, b \in A_{q}$, the function

$$
\frac{u \Delta\left(e^{u} ; q, a, b\right)}{e^{u / 2}}
$$

has a probabilistic distribution. This distribution (i) has mean $(N(q, b)-N(q, a)) / \phi(q)$, (ii) is symmetric with respect to its mean, and (iii) has a continuous density function.

Assume $a$ is a quadratic nonresidue modulo $q$ and $b$ is a quadratic residue. Then $N(q, b)-N(q, a)>0$. Let $f$ be the density function for the distribution of (1.3), that is,

$$
f(t)=\frac{d}{d t} \lim _{U \rightarrow \infty} \frac{1}{U} \operatorname{meas}\left\{0 \leqslant u \leqslant U: u e^{-u / 2} \Delta\left(e^{u} ; q, a, b\right) \leqslant t\right\} .
$$

We see from Theorem RS that

and from Theorem 2 that

$$
\delta(q, a, b)=\int_{0}^{\infty} f(t) d t>\frac{1}{2}
$$

$$
\delta_{2}(q, a, b)=\int_{-\infty}^{\frac{N(q, b)-N(q, a)}{2 \phi(q)}} f(t) d t
$$




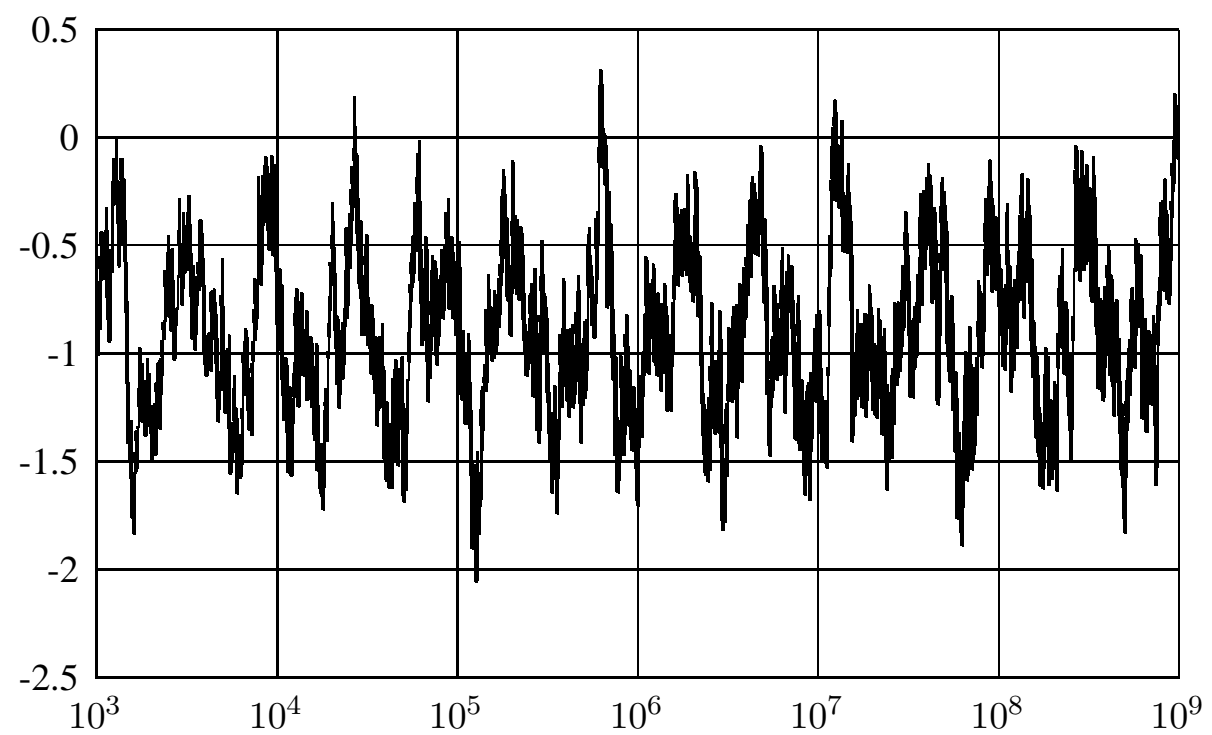

FIGURE 2. $\frac{\log x}{\sqrt{x} \log \log x} \Delta_{2}(x ; 4,3,1)$

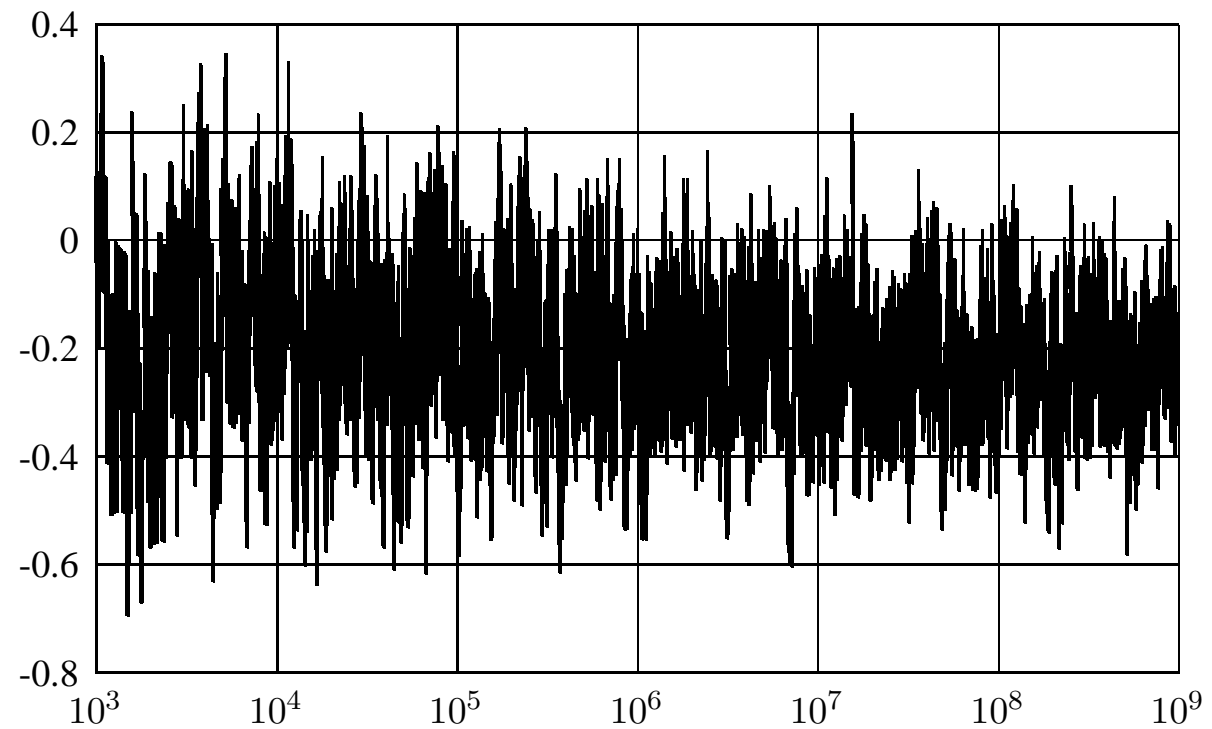

FIGURE 3. $\Sigma(x ; 4,3,1)$

from which Theorem 1 follows.

Theorem 2 also determines the joint distribution of any vector function

$$
\frac{u}{e^{u / 2} \log u}\left(\Delta_{2}\left(e^{u} ; q, a_{1}, b_{1}\right), \ldots, \Delta_{2}\left(e^{u} ; q, a_{r}, b_{r}\right)\right) .
$$

Theorem 3. If $f\left(x_{1}, \ldots, x_{r}\right)$ is the density function of

$$
\frac{u}{e^{u / 2}}\left(\Delta\left(e^{u} ; q, a_{1}, b_{1}\right), \ldots, \Delta\left(e^{u} ; q, a_{r}, b_{r}\right)\right),
$$


then the joint density function of (1.4) is

$$
f\left(\frac{N\left(q, b_{1}\right)-N\left(q, a_{1}\right)}{2 \phi(q)}-x_{1}, \ldots, \frac{N\left(q, b_{r}\right)-N\left(q, a_{r}\right)}{2 \phi(q)}-x_{r}\right) .
$$

1.3. Origin of Chebyshev's bias. From an analytic point of view ( $L$-functions), the weighted sum

$$
\Delta^{*}(x ; q, a, b)=\sum_{\substack{n \leqslant x \\ n \equiv a \bmod q}} \Lambda(n)-\sum_{\substack{n \leqslant x \\ n \equiv b \bmod q}} \Lambda(n),
$$

where $\Lambda$ is the von Mangoldt function, is more natural than (1.2). Expressing $\Delta^{*}(x ; q, a, b)$ in terms of sums over zeros of $L$-functions in the standard way ( $\S 19$ of $[\overline{\mathrm{Da}}]$ ), we obtain, on $\mathrm{ERH}_{q}$,

$$
e^{-u / 2} \phi(q) \Delta^{*}\left(e^{u} ; q, a, b\right)=-\sum_{\chi \in C(q)}(\bar{\chi}(a)-\bar{\chi}(b)) \sum_{\gamma} \frac{e^{i \gamma u}}{1 / 2+i \gamma}+O\left(u^{2} e^{-u / 2}\right),
$$

where $\gamma$ runs over imaginary parts of nontrivial zeros of $L(s, \chi)$ (counted with multiplicity). Hypothesis $\mathrm{GSH}_{q}$ implies, in particular, that $L(1 / 2, \chi) \neq 0$. Each summand $e^{i \gamma u} /(1 / 2+i \gamma)$ is thus a harmonic with mean zero as $u \rightarrow \infty$, and $\mathrm{GSH}_{q}$ implies that the harmonics behave independently. Hence, we expect that $e^{-u / 2} \phi(q) \Delta^{*}\left(e^{u} ; q, a, b\right)$ will behave like a mean zero random variable. On the other hand, the right side of (1.5) contains not only terms corresponding to prime $n$ but terms corresponding to powers of primes. Applying the prime number theorem for arithmetic progressions (1.1) to the terms $n=p^{2}$ in (1.5) gives

$$
\Delta^{*}(x ; q, a, b)=\sum_{\substack{p \leqslant x \\ p \equiv a \bmod q}} \log p-\sum_{\substack{p \leqslant x \\ p \equiv b \bmod q}} \log p+\frac{x^{1 / 2}}{\phi(q)}(N(q, a)-N(q, b))+O\left(x^{1 / 3}\right) .
$$

Hence, on $\mathrm{ERH}_{q}$ and $\mathrm{GSH}_{q}$, we expect the expression

$$
\frac{1}{\sqrt{x}}\left(\sum_{\substack{p \leqslant x \\ p \equiv a \bmod q}} \log p-\sum_{\substack{p \leqslant x \\ p \equiv b \bmod q}} \log p\right)
$$

to behave like a random variable with mean $(N(q, b)-N(q, a)) / \phi(q)$. Finally, the distribution of $\Delta(x ; q, a, b)$ is obtained from the distribution of (1.6) and partial summation.

1.4. Analyzing $\Delta_{2}(x ; q, a, b)$. A natural analog of $\Delta^{*}(x ; q, a, b)$ is

$$
\sum_{\substack{m n \leqslant x \\ m n \equiv a \bmod q}} \Lambda(m) \Lambda(n)-\sum_{\substack{m n \leqslant x \\ m n \equiv b \bmod q}} \Lambda(m) \Lambda(n) .
$$

As with $\Delta^{*}(x ; q, a, b)$, the expression in (1.7) can be easily written as a sum over zeros of $L$-functions plus a small error. The main problem now is that the principal summands, namely $\log p_{1} \log p_{2}$ for primes $p_{1}, p_{2}$, are very irregular as a function of $p_{1} p_{2}$, and thus estimates for $\Delta_{2}(x ; q, a, b)$ cannot be recovered by partial summation. We get around this problem using a double integration, a method which goes back to Landau [La, §88]. We have

$$
\begin{aligned}
\Delta_{2}(x ; q, a, b) & =\frac{1}{\phi(q)} \sum_{\chi \in C(q)}(\bar{\chi}(a)-\bar{\chi}(b)) \sum_{\substack{n=p_{1} p_{2} \leqslant x \\
p_{1} \leqslant p_{2}}} \chi(n) \\
& =\frac{1}{2 \phi(q)} \sum_{\chi \in C(q)}(\bar{\chi}(a)-\bar{\chi}(b)) \int_{0}^{\infty} \int_{0}^{\infty} G(x, u, v ; \chi) d u d v+O\left(\frac{\sqrt{x}}{\log x}\right),
\end{aligned}
$$

where

$$
G(x, u, v ; \chi)=\sum_{p_{1} p_{2} \leqslant x} \frac{\chi\left(p_{1} p_{2}\right) \log p_{1} \log p_{2}}{p_{1}^{u} p_{2}^{v}} .
$$


The related functions

$$
G^{*}(x, u, v ; \chi)=\sum_{m n \leqslant x} \frac{\chi(m n) \Lambda(m) \Lambda(n)}{m^{u} n^{v}}
$$

are more "natural" from an analytic point of view, being easily expressed in terms of zeros of Dirichlet $L$-functions. By the reasoning of the previous subsection, each $G^{*}(x, u, v ; \chi)$ is expected to be unbiased, the bias in $\Delta_{2}(x ; q, a, b)$ originating from the summands in $G^{*}(x, u, v ; \chi)$ where $m$ is not prime or $n$ is not prime.

1.5. A heuristic argument for the bias in $\Delta_{2}(x ; q, a, b)$. We conclude this introduction with a heuristic evaluation of the bias in $\Delta_{2}(x ; q, a, b)$, which originates from the difference between functions $G(x ; u, v ; \chi)$ and $G^{*}(x, u, v ; \chi)$. For simplicity of exposition, we'll concentrate on the special case $(q, a, b)=(4,3,1)$. In this case, the bias arises from terms $p_{1} p_{2}^{2}$ and $p_{1}^{2} p_{2}^{2}$ which appear in $G^{*}(x ; u, v ; \chi)$ but not in $G(x, u, v ; \chi)$. Let $\chi$ be the non-principal character modulo 4 , so that

$$
\frac{1}{2} \int_{0}^{\infty} \int_{0}^{\infty}\left(G^{*}(x, u, v ; \chi)-G(x, u, v ; \chi)\right) d u d v=\frac{1}{2} \sum_{\substack{p_{1}^{a} p_{2}^{b} \leqslant x \\ \max (a, b) \geqslant 2}} \frac{\chi\left(p_{1}^{a} p_{2}^{b}\right)}{a b}
$$

There are $O\left(x^{1 / 2} / \log x\right)$ terms with $\min (a, b) \geqslant 2$ and $\max (a, b) \geqslant 3$. By the prime number theorem and partial summation,

$$
\frac{1}{2} \sum_{p_{1}^{2} p_{2}^{2} \leqslant x} \frac{1}{4}=\frac{1}{8} \sum_{p \leqslant \sqrt{x}} \pi\left(\sqrt{x / p^{2}}\right) \sim \frac{x^{1 / 2} \log \log x}{2 \log x}
$$

Thus,

$$
\begin{aligned}
\Delta_{2}(x ; 4,3,1)=- & \frac{1}{2} \sum_{m n \leqslant x} \frac{\chi(m n) \Lambda(m) \Lambda(n)}{\log m \log n}-\left(\sum_{k=2}^{\infty} \frac{1}{k} \sum_{p_{1}^{k} \leqslant x} \chi\left(p_{1}^{k}\right) \Delta\left(x / p_{1}^{k} ; 4,3,1\right)\right) \\
& +\left(\frac{1}{2}+o(1)\right) \frac{x^{1 / 2} \log \log x}{\log x} .
\end{aligned}
$$

By Theorem RS, $\Delta(y ; 4,3,1)=y^{1 / 2} / \log y+E(y)$, where $E(y)$ oscillates with mean 0 . Thus,

$$
\sum_{k=2}^{\infty} \frac{1}{k} \sum_{p_{1}^{k} \leqslant x} \chi\left(p_{1}^{k}\right) \Delta\left(x / p_{1}^{k} ; 4,3,1\right)=\sum_{k=2}^{\infty} \frac{2}{k} \sum_{p_{1}^{k} \leqslant x} \chi\left(p_{1}^{k}\right) \frac{\sqrt{x / p_{1}^{k}}}{\log \left(x / p_{1}^{k}\right)}+E^{\prime}(x),
$$

where $E^{\prime}(x)$ is expected to oscillate with mean zero. The $k=2$ terms are

$$
\sum_{p_{1}^{2} \leqslant x} \frac{\sqrt{x / p_{1}^{2}}}{\log \left(x / p_{1}^{2}\right)} \sim \frac{\sqrt{x} \log \log x}{\log x}
$$

while the terms corresponding to $k \geqslant 3$ contribute

$$
\ll \sum_{k=3}^{\infty} \frac{1}{k} \sum_{p_{1}^{k} \leqslant x} \frac{\sqrt{x / p_{1}^{k}}}{\log \left(x / p_{1}^{k}\right)} \ll \frac{\sqrt{x}}{\log x} .
$$

Thus, we find that

$$
\Delta_{2}(x ; 4,3,1)=-\frac{1}{2} \sum_{m n \leqslant x} \frac{\chi(m n) \Lambda(m) \Lambda(n)}{\log m \log n}-\left(\frac{1}{2}+o(1)\right) \frac{x^{1 / 2} \log \log x}{\log x}+E^{\prime}(x) .
$$


1.6. Further problems. It is natural to consider the distribution, in arithmetic progressions, of numbers composed of exactly $k$ prime factors, where $k \geqslant 3$ is fixed. As with the cases $k=1$ and $k=2$, we expect there to be no bias if we count all numbers $p_{1}^{a_{1}} p_{2}^{a_{2}} \cdots p_{k}^{a_{k}}$ with weight $\left(a_{1} \cdots a_{k}\right)^{-1}$. If, however, we count terms which are the product of precisely $k$ primes (that is, numbers $p_{1}^{a_{1}} \cdots p_{j}^{a_{j}}$ with $a_{1}+\cdots+a_{j}=k$ ), then there will be a bias. Hudson has conjectured that the bias will be in the same direction as for primes when $k$ is odd, and in the opposite direction for even $k$. We conjecture that, in addition, the bias becomes less pronounced as $k$ increases.

\section{PRELIMINARIES}

With $\chi$ fixed, the letter $\gamma$, with or without subscripts, denotes the imaginary part of a zero of $L(s, \chi)$ inside the critical strip. In sums over $\gamma$, each term appears with its multiplicity $m(\gamma)$ unless we specify that we sum over distinct $\gamma$. Constants implied by $O-$ and $\ll-$ symbols depend only on $\chi$ (and hence, on $q$ ) unless additional dependence is indicated with a subscript. Let

$$
A(\chi)= \begin{cases}1 & \chi^{2}=\chi_{0} \\ 0 & \text { else }\end{cases}
$$

where $\chi_{0}$ is the principal character modulo $q$. That is, $A(\chi)=1$ if and only if $\chi$ is a real character. For $\chi \in C(q)$, define

$$
F(s, \chi)=\sum_{p} \frac{\chi(p) \log p}{p^{s}} .
$$

The following estimates are standard; see e.g. [Da, $\S 15,16]$.

Lemma 2.1. Let $\chi \in C(q)$, assume $E R H_{q}$ and fix $c>\frac{1}{3}$. Then $F(s, \chi)=-\frac{L^{\prime}}{L}(s, \chi)+A(\chi) \frac{\zeta^{\prime}}{\zeta}(2 s)+$ $H(s, \chi)$, where $H(s, \chi)$ is analytic and uniformly bounded in the half-plane $\Re s \geqslant c$.

Lemma 2.2. Let $\chi$ be a Dirichlet character modulo $q$. Let $N(T, \chi)$ denote the number of zeros of $L(s, \chi)$ with $0<\Re s<1$ and $|\Im s|<T$. Then

(1) $N(T, \chi)=O(T \log (q T))$ for $T \geqslant 1$.

(2) $N(T, \chi)-N(T-1, \chi)=O(\log (q T))$ for $T \geqslant 1$.

(3) Uniformly for $s=\sigma+i t$ and $\sigma \geqslant-1$,

$$
\frac{L^{\prime}(s, \chi)}{L(s, \chi)}=\sum_{|\gamma-t|<1} \frac{1}{s-\rho}+O(\log q(|t|+2)) .
$$

(4) $-\frac{\zeta^{\prime}}{\zeta}(\sigma)=\frac{1}{\sigma-1}+O(1)$ uniformly for $\sigma \geqslant \frac{1}{2}, \sigma \neq 1$.

(5) $\left|\frac{\zeta^{\prime}}{\zeta}(\sigma+i T)\right| \leqslant-\frac{\zeta^{\prime}}{\zeta}(\sigma)$ for $\sigma>1$.

For a suitably small, fixed $\delta>0$, we say that a number $T \geqslant 2$ is admissible if for all $\chi \in C(q) \cup\left\{\chi_{0}\right\}$ and all zeros $\frac{1}{2}+i \gamma$ of $L(s, \chi),|\gamma-T| \geqslant \delta(\log T)^{-1}$. By Lemma 2.2, we can choose $\delta$ small enough, depending on $q$, so that there is an admissible $T$ in $[U, U+1]$ for all $U \geqslant 2$. From Lemma 2.2 we obtain

Lemma 2.3. Uniformly for $\sigma \geqslant \frac{2}{5}$ and admissible $T \geqslant 2$,

$$
|F(\sigma+i T, \chi)|=O\left(\log ^{2} T\right) .
$$

Lemma 2.4. Fix $\chi \in C(q)$ and assume $L\left(\frac{1}{2}, \chi\right) \neq 0$. For $A \geqslant 0$ and real $k \geqslant 0$,

$$
\sum_{\substack{\left|\gamma_{1}\right|,\left|\gamma_{2}\right| \geqslant A \\\left|\gamma_{1}-\gamma_{2}\right| \geqslant 1}} \frac{\log ^{k}\left(\left|\gamma_{1}\right|+3\right) \log ^{k}\left(\left|\gamma_{2}\right|+3\right)}{\left|\gamma_{1}\right|\left|\gamma_{2}\right|\left|\gamma_{1}-\gamma_{2}\right|} \ll_{k} \frac{(\log (A+3))^{2 k+3}}{A+1} .
$$


Proof. The sum in question is at most twice the sum of terms with $\left|\gamma_{2}\right| \geqslant\left|\gamma_{1}\right|$, which is

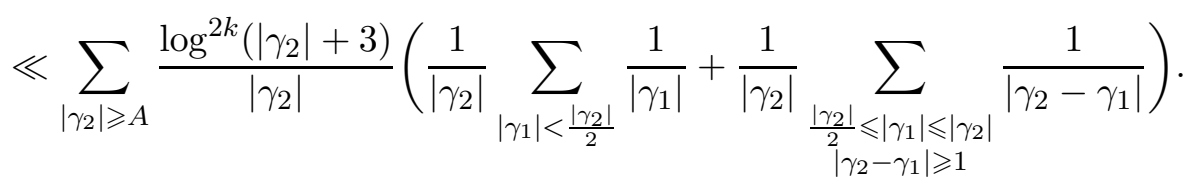

By Lemma 2.2 (1), the two sums over $\gamma_{1}$ are $O\left(\log ^{2}\left(\left|\gamma_{2}\right|+3\right)\right)$. A further application of Lemma 2.2 (1) completes the proof.

We conclude this section with a truncated version of the Perron formula for $G(x, u, v ; \chi)$.

Lemma 2.5. Uniformly for $x \leqslant T \leqslant 2 x^{2}, x \geqslant 2, u \geqslant 0$ and $v \geqslant 0$, we have

$$
G(x, u, v ; \chi)=\frac{1}{2 \pi i} \int_{c-i T}^{c+i T} F(s+u, \chi) F(s+v, \chi) \frac{x^{s}}{s} d s+O\left(\log ^{3} x\right),
$$

where $c=1+\frac{1}{\log x}$.

Proof. For $\Re s>1$, we have

$$
F(s+u, \chi) F(s+v, \chi)=\sum_{n=1}^{\infty} f(n) n^{-s}, \quad f(n)=\sum_{p_{1} p_{2}=n} \frac{\chi\left(p_{1} p_{2}\right) \log p_{1} \log p_{2}}{p_{1}^{u} p_{2}^{v}} .
$$

Using the trivial estimate $|f(n)| \leqslant \log ^{2} n$ and a standard argument [Da, $\S 17$, (3) and (5)], we obtain the desired bounds.

\section{OUtLine OF THE PROOF OF THEOREM 2} Let

Throughout the remainder of this paper, fix $q$, assume $\mathrm{ERH}_{q}$ and that $L\left(\frac{1}{2}, \chi\right) \neq 0$ for each $\chi \in C(q)$.

$$
\varepsilon=\frac{1}{100}
$$

We next define a function $T(x)$ as follows. For each positive integer $n$, let $T_{n}$ be an admissible value of $T$ satisfying $\exp \left(2^{n+1}\right) \leqslant T_{n} \leqslant \exp \left(2^{n+1}\right)+1$ and set $T(x)=T_{n}$ for $\exp \left(2^{n}\right)<x \leqslant \exp \left(2^{n+1}\right)$. In particular, we have

$$
x \leqslant T(x) \leqslant 2 x^{2} \quad\left(x \geqslant e^{2}\right) .
$$

Our first task is to express the double integrals in (1.8) in terms of sums over zeros of $L(s, \chi)$. This is proved in Section 4

Lemma 3.1. Let $\chi \in C(q)$ and let $T=T(x)$. Then

$$
\begin{aligned}
x^{-1 / 2} \int_{0}^{\infty} & \int_{0}^{\infty} G(x, u, v ; \chi) d u d v \\
& =2 \int_{0}^{2 \varepsilon} \int_{0}^{2 \varepsilon} \sum_{|\gamma| \leqslant T} \frac{F\left(\frac{1}{2}+u-v+i \gamma, \chi\right) x^{-v+i \gamma}}{\frac{1}{2}-v+i \gamma} d u d v+\frac{A(\chi) \log \log x+\Sigma_{1}(x ; \chi)+O(1)}{\log x},
\end{aligned}
$$

where $\int_{1}^{Y}\left|\Sigma_{1}\left(e^{y} ; \chi\right)\right|^{2} d y=O(Y)$.

The aggregate of terms $A(\chi) \log \log x / \log x$ account for the bias for products of two primes. As with the Chebyshev bias for primes, these terms arise from poles of $F(s)$ at $s=\frac{1}{2}$ when $A(\chi)=1$ (see Lemma2.1) and correspond to the contribution to $F(s)$ from squares of primes. The double integral on the right side in Lemma 3.1 is complicated to analyze. In Section 5 we prove the following. 
Lemma 3.2. Let $\chi \in C(q)$. Let $n$ be a positive integer, $2^{n}<\log x \leqslant 2^{n+1}$ and $T=T(x)$. Then

$$
\begin{aligned}
2 \int_{0}^{2 \varepsilon} \int_{0}^{2 \varepsilon} & \sum_{|\gamma| \leqslant T} \frac{F\left(\frac{1}{2}+u-v+i \gamma, \chi\right) x^{-v+i \gamma}}{\frac{1}{2}-v+i \gamma} d u d v=\frac{\Sigma_{2}(x ; \chi)}{\log x} \\
& +2 \sum_{\substack{|\gamma| \leqslant T \\
\gamma \text { distinct }}} m^{2}(\gamma) x^{i \gamma}\left(\frac{1}{2}+i \gamma\right) \int_{0}^{2 \varepsilon-2^{-n}} \frac{x^{-v}}{\frac{1}{2}-v+i \gamma} \int_{v+2^{-n}}^{2 \varepsilon} \frac{d u}{(u-v)\left(\frac{1}{2}-u+i \gamma\right)} d v
\end{aligned}
$$

where $\int_{1}^{Y}\left|\Sigma_{2}\left(e^{y} ; \chi\right)\right|^{2} d y=o\left(Y \log ^{2} Y\right)$.

The terms on the right in Lemma 3.2 with small $|\gamma|$ will give the main term, and terms with larger $|\gamma|$ are considered as error terms. The next lemma is proved in Section 6

Lemma 3.3. Let $\chi \in C(q)$. Let $n$ be a positive integer, $2^{n}<\log x \leqslant 2^{n+1}, T=T(x)$ and $2 \leqslant T_{0} \leqslant T$. Then

$$
\begin{aligned}
& 2 \sum_{\substack{|\gamma| \leqslant T \\
\gamma \text { distinct }}} m^{2}(\gamma) x^{i \gamma}\left(\frac{1}{2}+i \gamma\right) \int_{0}^{2 \varepsilon-2^{-n}} \frac{x^{-v}}{\frac{1}{2}-v+i \gamma} \int_{v+2^{-n}}^{2 \varepsilon} \frac{d u}{(u-v)\left(\frac{1}{2}-u+i \gamma\right)} d v \\
& \quad=\frac{2 \log \log x}{\log x} \sum_{\substack{|\gamma| \leqslant T_{0} \\
\gamma \text { distinct }}} \frac{m^{2}(\gamma) x^{i \gamma}}{1 / 2+i \gamma}+O\left(\frac{\log ^{3} T_{0}}{\log x}\right)+\frac{\Sigma_{3}\left(x, T_{0} ; \chi\right)}{\log x},
\end{aligned}
$$

where

$$
\frac{1}{Y} \int_{1}^{Y}\left|\Sigma_{3}\left(e^{y}, T_{0} ; \chi\right)\right|^{2} d y \ll \frac{\log ^{5} T_{0}}{T_{0}} \log ^{2} Y .
$$

Combining Lemmas 3.1, 3.2 and 3.3 with (1.8) yields (for fixed, large $T_{0}$ )

$$
\begin{aligned}
\Delta_{2}(x ; q, a, b)=\frac{\sqrt{x}}{2 \phi(q)} \sum_{\chi \in C(q)}(\bar{\chi}(a)-\bar{\chi}(b)) & {\left[\frac{\log \log x}{\log x}\left(A(\chi)+2 \sum_{\substack{|\gamma| \leqslant T_{0} \\
\gamma \text { distinct }}} \frac{m^{2}(\gamma) x^{i \gamma}}{1 / 2+i \gamma}\right)\right.} \\
& \left.+\frac{\Sigma_{1}(x ; \chi)+\Sigma_{2}(x ; \chi)+\Sigma_{3}\left(x, T_{0} ; \chi\right)+O\left(\log ^{3} T_{0}\right)}{\log x}\right],
\end{aligned}
$$

where

$$
\lim _{T_{0} \rightarrow \infty}\left(\limsup _{Y \rightarrow \infty} \frac{1}{Y \log ^{2} Y} \sum_{\chi \in C(q)} \int_{1}^{Y}\left|\Sigma_{1}\left(e^{y} ; \chi\right)+\Sigma_{2}\left(e^{y} ; \chi\right)+\Sigma_{3}\left(e^{y} ; T_{0} ; \chi\right)\right|^{2} d y\right)=0 .
$$

On the other hand (cf. [RS]),

$$
\Delta(x ; q, a, b)=\frac{\sqrt{x}}{\log x}\left(\frac{N(q, b)-N(q, a)}{\phi(q)}-\sum_{\chi \in C(q)}(\bar{\chi}(a)-\bar{\chi}(b)) \sum_{|\gamma| \leqslant T_{0}} \frac{x^{i \gamma}}{1 / 2+i \gamma}+\Sigma_{4}\left(x ; T_{0}\right)\right),
$$

where

$$
\lim _{T_{0} \rightarrow \infty}\left(\limsup _{Y \rightarrow \infty} Y^{-1} \int_{1}^{Y}\left|\Sigma_{4}\left(e^{y} ; T_{0}\right)\right|^{2} d y\right)=0 .
$$

Now assume $m(\gamma)=1$ for all $\gamma$, and note that

$$
\sum_{\chi \in C(q)}(\bar{\chi}(a)-\bar{\chi}(b)) A(\chi)=N(q, a)-N(q, b) .
$$

Letting $T_{0} \rightarrow \infty$ finishes the proof of Theorem 2 


\section{PROOF OF LEMMA 3.1}

Assume $\mathrm{ERH}_{q}$ throughout. We first estimate $G(x, u, v ; \chi)$ for different ranges of $u, v$.

Lemma 4.1. Let $\chi \in C(q), \chi \neq \chi_{0}$. For $x \geqslant 4$, the following hold:

(1) For $u, v \geqslant \varepsilon, G(x, u, v ; \chi) \ll x^{\frac{1}{2}-\frac{\varepsilon}{2}} \log ^{5} x$.

(2) For $u \geqslant 2 \varepsilon, v \leqslant \varepsilon$ and $T=T(x)$,

$$
\begin{gathered}
x^{-1 / 2} G(x, u, v ; \chi)=\sum_{|\gamma| \leqslant T} \frac{F\left(\frac{1}{2}+u-v+i \gamma, \chi\right) x^{-v+i \gamma}}{\frac{1}{2}-v+i \gamma}-A(\chi) \frac{F\left(\frac{1}{2}+u-v, \chi\right) x^{-v}}{1-2 v} \\
+O\left(x^{-\frac{3 \varepsilon}{2}} \log ^{5} x\right) .
\end{gathered}
$$

(3) For $u \leqslant 2 \varepsilon, v \leqslant 2 \varepsilon, u \neq v$ and $T=T(x)$,

$$
\begin{aligned}
x^{-1 / 2} G(x, u, v ; \chi)= & \sum_{|\gamma| \leqslant T} \frac{F\left(\frac{1}{2}+u-v+i \gamma, \chi\right) x^{-v+i \gamma}}{\frac{1}{2}-v+i \gamma}+\frac{F\left(\frac{1}{2}-u+v+i \gamma, \chi\right) x^{-u+i \gamma}}{\frac{1}{2}-u+i \gamma} \\
& -A(\chi)\left(\frac{F\left(\frac{1}{2}+u-v, \chi\right) x^{-v}}{1-2 v}+\frac{F\left(\frac{1}{2}-u+v, \chi\right) x^{-u}}{1-2 u}\right)+O\left(x^{-3 \varepsilon} \log ^{5} x\right) .
\end{aligned}
$$

Proof. Assume $u \geqslant \varepsilon$ and $v \geqslant \varepsilon$. Start with the approximation of $G(x, u, v ; \chi)$ given by Lemma 2.5, then deform the segment of integration to the contour consisting of three straight segments connecting $c-i T$, $b-i T, b+i T$ and $c+i T$, where $b=\frac{1}{2}-\frac{\varepsilon}{2}$ and $T=T(x)$. The rectangle formed by the new and old contours does not contain any poles of $F(s+u, \chi) F(s+v, \chi) s^{-1}$. On the three new segments, by Lemmas 2.1, 2.2 and 2.3, we have $|F(s+u, \chi) F(s+v, \chi)| \ll \log ^{4} T$. Hence the integral of $F(s+u, \chi) F(s+v, \chi) x^{s} s^{-1}$ over the three segments is

$$
\ll\left(\log ^{4} x\right)\left(\int_{b}^{c} \frac{x^{\sigma}}{|\sigma+i T|} d \sigma+\int_{-T}^{T} \frac{x^{b}}{|b+i t|} d t\right) \ll x^{b} \log ^{5} x .
$$

This proves (1).

We now consider the case $v \leqslant \varepsilon$ and $u \geqslant 2 \varepsilon$. We set $b=\frac{1}{2}-\frac{3 \varepsilon}{2}$ and deform the contour of integration as in the previous case. Since $u+b \geqslant \frac{1}{2}+\frac{\varepsilon}{2}$ and $v+b \leqslant \frac{1}{2}-\frac{\varepsilon}{2}$, we have by Lemma 2.3 that $\mid F(s+$ $u, \chi) F(s+v, \chi) \mid \ll \log ^{4} T \ll \log ^{4} x$ on all three new segments. As in the proof of (1), the integral over the new contour is $\ll x^{b} \log ^{5} x$. We pick up residue terms from poles of $F(s+v, \chi)$ inside the rectangle coming from the nontrivial zeros of $L(s, \chi)$, plus a pole at $s=\frac{1}{2}-v$ from the $\frac{\zeta^{\prime}}{\zeta}(2 s+2 v)$ term if $\chi^{2}=\chi_{0}$. The sum of the residues is

$$
\sum_{|\gamma| \leqslant T} \frac{F\left(\frac{1}{2}+u-v+i \gamma, \chi\right) x^{\frac{1}{2}-v+i \gamma}}{\frac{1}{2}-v+i \gamma}-A(\chi) \frac{F\left(\frac{1}{2}+u-v, \chi\right) x^{\frac{1}{2}-v}}{1-2 v},
$$

and (2) follows.

Finally, consider the case $0 \leqslant u, v \leqslant 2 \varepsilon$. Let $b=\frac{1}{2}-3 \varepsilon$ and deform the contour as in the previous cases. As before, the integral over the new contour is $O\left(x^{b} \log ^{5} x\right)$. This time, we pick up residues from poles of both $F(s+u, \chi)$ and $F(s+v, \chi)$. The sum of the residues is

$$
\begin{gathered}
\sum_{|\gamma| \leqslant T}\left(\frac{F\left(\frac{1}{2}+u-v+i \gamma, \chi\right) x^{\frac{1}{2}-v+i \gamma}}{\frac{1}{2}-v+i \gamma}+\frac{F\left(\frac{1}{2}-u+v+i \gamma, \chi\right) x^{\frac{1}{2}-u+i \gamma}}{\frac{1}{2}-u+i \gamma}\right) \\
-A(\chi)\left(\frac{F\left(\frac{1}{2}+u-v, \chi\right) x^{\frac{1}{2}-v}}{1-2 v}+\frac{F\left(\frac{1}{2}-u+v, \chi\right) x^{\frac{1}{2}-u}}{1-2 u}\right),
\end{gathered}
$$

and (3) follows. 
Proof of Lemma 3.1. Begin with

$$
\int_{0}^{\infty} \int_{0}^{\infty} G(x, u, v ; \chi) d u d v=I_{1}+I_{2}+2 I_{3}+I_{4}
$$

where $I_{1}$ is the integral over $\max (u, v) \geqslant \log x, I_{2}$ is the integral over $2 \varepsilon \leqslant \max (u, v) \leqslant \log x$ and $\min (u, v) \geqslant \varepsilon, I_{3}$ is the integral over $0 \leqslant v \leqslant \varepsilon, 2 \varepsilon \leqslant u \leqslant \log x$, and $I_{4}$ is the integral over $0 \leqslant u, v \leqslant 2 \varepsilon$. For $\max (u, v) \geqslant \log x$,

$$
|G(x, u, v ; \chi)| \leqslant \sum_{p \leqslant x} \frac{\log p}{p^{u}} \sum_{p \leqslant x} \frac{\log q}{q^{v}} \ll \frac{x}{2^{\max (u, v)}},
$$

whence $I_{1} \ll x^{1-\log 2}$. By Lemma $4.1(1), I_{2} \ll x^{1 / 2-\varepsilon / 2} \log ^{7} x$.

By Lemma4.1(2),

$$
\begin{aligned}
I_{3}=x^{1 / 2} \int_{0}^{\varepsilon} \int_{2 \varepsilon}^{\log x} \sum_{|\gamma| \leqslant T} \frac{F\left(\frac{1}{2}+u-v+i \gamma, \chi\right) x^{-v+i \gamma}}{\frac{1}{2}-v+i \gamma}-A(\chi) \frac{F\left(\frac{1}{2}+u-v, \chi\right) x^{-v}}{1-2 v} d u d v \\
\quad+O\left(x^{1 / 2-\frac{3 \varepsilon}{2}} \log ^{6} x\right) .
\end{aligned}
$$

By Lemmas 2.2 and 2.3 ,

$$
\int_{0}^{\varepsilon} \int_{2 \varepsilon}^{\log x} \frac{F\left(\frac{1}{2}+u-v, \chi\right) x^{-v}}{1-2 v} d u d v \ll \int_{0}^{\varepsilon} x^{-v} d v \ll \frac{1}{\log x}
$$

Let

$$
\Sigma_{1}(x)=(\log x) \int_{0}^{\varepsilon} \int_{2 \varepsilon}^{\log x} \sum_{0<|\gamma|<T} \frac{F\left(\frac{1}{2}+u-v+i \gamma, \chi\right) x^{-v+i \gamma}}{\frac{1}{2}-v+i \gamma} d u d v
$$

Since $\frac{1}{2}+u-v \geqslant \frac{1}{2}+\varepsilon$ for $0 \leqslant v \leqslant \varepsilon$ and $2 \varepsilon \leqslant u \leqslant \log x$, by Lemmas 2.1, 2.2, and 2.3,

$$
F\left(\frac{1}{2}+u-v+i \gamma, \chi\right)=-\frac{L^{\prime}}{L}\left(\frac{1}{2}+u-v+i \gamma, \chi\right)+O(1) \ll \log (|\gamma|+3) .
$$

We also have $F(1 / 2+u-v+i \gamma, \chi) \ll 2^{-u}$ for $u \geqslant 2$. Thus, for positive integers $n$,

$$
\begin{aligned}
\int_{2^{n}}^{2^{n+1}}\left|\Sigma_{1}\left(e^{y}\right)\right|^{2} d y \ll 2^{2 n} & \sum_{\left|\gamma_{1}\right|,\left|\gamma_{2}\right| \leqslant T} \frac{\log \left(\left|\gamma_{1}\right|+3\right) \log \left(\left|\gamma_{2}\right|+3\right)}{\left|\gamma_{1} \gamma_{2}\right|} \\
& \times \int_{0}^{\varepsilon} \int_{0}^{\varepsilon}\left|\int_{2^{n}}^{2^{n+1}} e^{y\left(-v_{1}+i \gamma_{1}-v_{2}-i \gamma_{2}\right)} d y\right| d v_{1} d v_{2} .
\end{aligned}
$$

The summands with $\left|\gamma_{1}-\gamma_{2}\right|<1$ contribute, by Lemma 2.2,

$$
\begin{aligned}
& \ll 2^{2 n} \sum_{\substack{\left|\gamma_{1}\right|,\left|\gamma_{2}\right| \leqslant T \\
\left|\gamma_{1}-\gamma_{2}\right|<1}} \frac{\log \left(\left|\gamma_{1}\right|+3\right) \log \left(\left|\gamma_{2}\right|+3\right)}{\left|\gamma_{1}\right|\left|\gamma_{2}\right|} \int_{2^{n}}^{2^{n+1}}\left(\int_{0}^{\varepsilon} e^{-v y} d v\right)^{2} d y \\
& \ll 2^{n} \sum_{|\gamma| \leqslant T} \frac{\log ^{3}(|\gamma|+3)}{|\gamma|^{2}} \ll 2^{n} .
\end{aligned}
$$

The summands with $\left|\gamma_{1}-\gamma_{2}\right| \geqslant 1$ contribute, by Lemma 2.4

$$
\ll \sum_{\substack{\left|\gamma_{1}\right|,\left|\gamma_{2}\right|<T \\\left|\gamma_{1}-\gamma_{2}\right| \geqslant 1}} \frac{2^{2 n} \log \left(\left|\gamma_{1}\right|+3\right) \log \left(\left|\gamma_{2}\right|+3\right)}{\left|\gamma_{1}\right|\left|\gamma_{2}\right|\left|\gamma_{1}-\gamma_{2}\right|}\left(\int_{0}^{\varepsilon} e^{-v 2^{n}} d v\right)^{2} \ll 1 .
$$

Thus, $\int_{2^{n}}^{2^{n+1}}\left|\Sigma_{1}\left(e^{y}\right)\right|^{2} d y=O\left(2^{n}\right)$. Summing over $n \leqslant \frac{\log Y}{\log 2}+1$ yields $\int_{1}^{Y}\left|\Sigma_{1}\left(e^{y}\right)\right|^{2} d y=O(Y)$. 
Finally, using Lemma4.1(3) gives

$$
\begin{aligned}
I_{4}= & x^{1 / 2} \int_{0}^{2 \varepsilon} \int_{0}^{2 \varepsilon} \sum_{|\gamma| \leqslant T} \frac{F\left(\frac{1}{2}+u-v+i \gamma, \chi\right) x^{-v+i \gamma}}{\frac{1}{2}-v+i \gamma}+\frac{F\left(\frac{1}{2}-u+v+i \gamma, \chi\right) x^{-u+i \gamma}}{\frac{1}{2}-u+i \gamma} \\
& -A(\chi)\left(\frac{F\left(\frac{1}{2}+u-v, \chi\right) x^{-v}}{1-2 v}+\frac{F\left(\frac{1}{2}-u+v, \chi\right) x^{-u}}{1-2 u}\right) d u d v+O\left(x^{\frac{1}{2}-3 \varepsilon} \log ^{3} x\right) .
\end{aligned}
$$

Now assume $\chi^{2}=\chi_{0}$. We will show that

$$
-\int_{0}^{2 \varepsilon} \int_{0}^{2 \varepsilon} \frac{F\left(\frac{1}{2}+u-v, \chi\right) x^{-v}}{1-2 v}+\frac{F\left(\frac{1}{2}-u+v, \chi\right) x^{-u}}{1-2 u} d u d v=\frac{\log \log x+O(1)}{\log x} .
$$

Together with (4.1), (4.2) and (4.3), this completes the proof of Lemma 3.1

Note that $F\left(\frac{1}{2}+w\right)=-\frac{1}{2 w}+O(1)$ by Lemmas 2.1 and 2.3. Replacing $x$ with $e^{y}$, the left side of (4.4) is

$$
=\frac{1}{2} \int_{0}^{2 \varepsilon} \int_{0}^{2 \varepsilon} \frac{e^{-y v}}{(u-v)(1-2 v)}+\frac{e^{-y u}}{(v-u)(1-2 u)} d u d v+O\left(\int_{0}^{2 \varepsilon} \int_{0}^{2 \varepsilon} e^{-y v} d u d v\right) .
$$

The error term above is $O(1 / y)$. In the main term, when $|u-v|<1 / y$, the integrand is $O\left(y e^{-v y}\right)$ and the corresponding part of the double integral is $O(1 / y)$. When $u \geqslant v+1 / y$, the integrand is

$$
\frac{e^{-v y}}{u-v}+O\left(\frac{v e^{-v y}+e^{-u y}}{u-v}\right)
$$

and the corresponding part of the double integral is

$$
\int_{0}^{2 \varepsilon} e^{-v y} \log \left(\frac{y}{2 \varepsilon-v}\right) d v+O\left(\frac{1}{y}\right)=\frac{\log y+O(1)}{y} .
$$

The contribution from $u \leqslant v-1 / y$ is, by symmetry, also $\frac{\log y+O(1)}{y}$. The asymptotic (4.4) follows.

\section{Proof of Lemma 3.2}

Lemma 5.1. Uniformly for $y \geqslant 1,0<|\xi| \leqslant 1,|w| \geqslant \frac{1}{2}$ and $a \geqslant 0$ we have

$$
\left|\int_{0}^{2 \varepsilon} \int_{0}^{2 \varepsilon} \frac{v^{a} e^{-v y}}{(u-v+i \xi)(w-v)} d u d v\right| \ll \frac{(4 \varepsilon)^{a} \log \min \left(2 y, \frac{2}{|\xi|}\right)}{y|w|} .
$$

Proof. Let $I$ denote the double integral in the Lemma. If $|\xi| \geqslant \frac{1}{y}$, then

$$
\begin{aligned}
I & \ll \frac{1}{|w|} \int_{0}^{2 \varepsilon} v^{a} e^{-v y} \int_{0}^{2 \varepsilon} \min \left(\frac{1}{|u-v|}, \frac{1}{|\xi|}\right) d u d v \\
& \ll \frac{(2 \varepsilon)^{a}}{|w|}\left(1+\log \frac{2}{|\xi|}\right) \int_{0}^{2 \varepsilon} e^{-v y} d v \ll \frac{(2 \varepsilon)^{a} \log \left(\frac{2}{|\xi|}\right)}{y|w|} .
\end{aligned}
$$

If $|\xi|<\frac{1}{y}$, let $I=I_{1}+I_{2}+I_{3}$, where $I_{1}$ is the part of $I$ coming from $|u-v| \leqslant|\xi|, I_{2}$ is the part of $I$ coming from $|\xi|<|u-v| \leqslant \frac{1}{y}$, and $I_{3}$ is the part of $I$ coming from $|u-v|>\frac{1}{y}$. We have

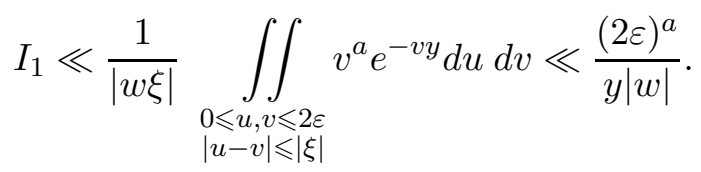

and

$$
I_{3} \ll \frac{(2 \varepsilon)^{a}}{|w|} \iint_{\substack{0 \leqslant u, v \leqslant 2 \varepsilon \\|u-v| \geqslant \frac{1}{y}}} \frac{e^{-v y}}{|u-v|} d u d v \ll \frac{(2 \varepsilon)^{a}}{|w|} \int_{0}^{2 \varepsilon} e^{-v y}(\log y+1) d v \ll \frac{(2 \varepsilon)^{a} \log (2 y)}{y|w|} .
$$


By symmetry,

$$
I_{2}=\frac{1}{2} \iint_{|\xi|<|u-v| \leqslant 1 / y} \frac{v^{a} e^{-v y}}{(u-v+i \xi)(w-v)}+\frac{u^{a} e^{-u y}}{(v-u+i \xi)(w-u)} d u d v .
$$

Since, $\left|u^{a}-v^{a}\right| \leqslant a|u-v|(2 \varepsilon)^{a-1}$,

$$
\begin{aligned}
u^{a} e^{-u y}-v^{a} e^{-v y} & =e^{-v y} v^{a}\left(e^{(v-u) y}-1\right)+e^{-v y}\left(u^{a}-v^{a}\right) e^{(v-u) y} \\
& \ll e^{-v y} y|u-v|(4 \varepsilon)^{a} .
\end{aligned}
$$

We deduce that

$$
\begin{aligned}
I_{2} & =\iint_{\substack{0 \leqslant u, v \leqslant 2 \varepsilon \\
|\xi|<|u-v| \leqslant 1 / y}} \frac{(w-u)(u-v)\left(u^{a} e^{-u y}-v^{a} e^{-v y}\right)+u^{a} e^{-u y}(u-v)^{2}+O\left(|\xi w|(2 \varepsilon)^{a} e^{-v y}\right)}{2(u-v+i \xi)(v-u+i \xi)(w-u)(w-v)} d u d v \\
& \ll \frac{(4 \varepsilon)^{a}}{|w|} \underset{\substack{0 \leqslant u, v \leqslant 2 \varepsilon \\
|\xi|<|u-v| \leqslant 1 / y}}{\int} y e^{-v y}+\frac{|\xi| e^{-v y}}{|u-v|^{2}} d u d v \ll \frac{(4 \varepsilon)^{a}}{y|w|} .
\end{aligned}
$$

Proof of Lemma 3.2 Let $y=\log x$. We first note by Lemmas 2.1 and 2.2,

$$
F\left(\frac{1}{2}+u-v+i \gamma, \chi\right)=\frac{m(\gamma)}{u-v}+R(\gamma, u-v)+R^{\prime}(\gamma, u-v)
$$

where

$$
R(\gamma, w)=\sum_{0<\left|\gamma^{\prime}-\gamma\right| \leqslant 1} \frac{1}{w+i\left(\gamma-\gamma^{\prime}\right)}, \quad R^{\prime}(\gamma, u-v)=O(\log (|\gamma|+3)) .
$$

Then, the double integral in Lemma 3.2 is

$$
=\sum_{i=1}^{4} \Sigma_{2, i}(y)+2 \sum_{\substack{|\gamma| \leqslant T \\ \gamma \text { distinct }}} m^{2}(\gamma) e^{i y \gamma}\left(\frac{1}{2}+i \gamma\right) \int_{0}^{2 \varepsilon-2^{-n}} \frac{e^{-y v}}{\frac{1}{2}-v+i \gamma} \int_{v+2^{-n}}^{2 \varepsilon} \frac{d u}{(u-v)\left(\frac{1}{2}-u+i \gamma\right)} d v
$$

where

$$
\begin{aligned}
& \Sigma_{2,1}(y)=2 \int_{0}^{2 \varepsilon} \int_{0}^{2 \varepsilon} \sum_{|\gamma| \leqslant T} \frac{R(\gamma, u-v) e^{y(-v+i \gamma)}}{\frac{1}{2}-v+i \gamma} d u d v \\
& \Sigma_{2,2}(y)=2 \int_{0}^{2 \varepsilon} \int_{0}^{2 \varepsilon} \frac{R^{\prime}(\gamma, u-v) e^{y(-v+i \gamma)}}{\frac{1}{2}-v+i \gamma} d u d v \\
& \Sigma_{2,3}(y)=\sum_{\substack{|\gamma| \leqslant T \\
\gamma \text { distinct }}} m^{2}(\gamma) e^{i y \gamma}\left(\frac{1}{2}+i \gamma\right) \int_{\substack{0 \leqslant u, v \leqslant 2 \varepsilon \\
|u-v| \leqslant 2^{-n}}} \frac{e^{-y v}-e^{-u y}}{(u-v)\left(\frac{1}{2}-v+i \gamma\right)\left(\frac{1}{2}-u+i \gamma\right)} d v d u \\
& \Sigma_{2,4}(y)=2 \sum_{\substack{|\gamma| \leqslant T \\
\gamma \text { distinct }}} m^{2}(\gamma) e^{i y \gamma}\left(\frac{1}{2}+i \gamma\right) \int_{2^{-n}}^{2 \varepsilon} \int_{0}^{v-2^{-n}} \frac{e^{-y v}}{(u-v)\left(\frac{1}{2}-v+i \gamma\right)\left(\frac{1}{2}-u+i \gamma\right)} d u d v .
\end{aligned}
$$

We show that $\sum_{j=1}^{4} \Sigma_{2, j}(y)$ is small in mean square. Note that for $2^{n}<y \leqslant 2^{n+1}, T=T\left(e^{y}\right)$ is constant. Also, by Lemma 2.2, we have

$$
m(\gamma) \ll \log (|\gamma|+3) .
$$


First, by Lemmas 2.2 and 2.4

$$
\begin{gathered}
\int_{2^{n}}^{2^{n+1}}\left|\Sigma_{2,2}(y)\right|^{2} d y=4 \iiint \int_{[0,2 \varepsilon]^{4}} \sum_{\substack{\left|\gamma_{1}\right| \leqslant T \\
\left|\gamma_{2}\right| \leqslant T}} \frac{R^{\prime}\left(\gamma_{1}, u_{1}-v_{1}\right) \overline{R^{\prime}\left(\gamma_{2}, u_{2}-v_{2}\right)}}{\left(\frac{1}{2}-v_{1}+i \gamma_{1}\right)\left(\frac{1}{2}-v_{2}-i \gamma_{2}\right)} \\
\quad \times \int_{2^{n}}^{2^{n+1}} e^{y\left(-v_{1}-v_{2}+i \gamma_{1}-i \gamma_{2}\right)} d y d u_{j} d v_{j} \\
\ll \sum_{\left|\gamma_{1}-\gamma_{2}\right|>1} \frac{\log \left(\left|\gamma_{1}\right|+3\right) \log \left(\left|\gamma_{2}\right|+3\right)}{\left|\gamma_{1} \gamma_{2}\right| \cdot\left|\gamma_{1}-\gamma_{2}\right|} \iiint \int_{[0,2 \varepsilon]^{4}} e^{-2^{n}\left(v_{1}+v_{2}\right)} d u_{j} d v_{j} \\
+\sum_{\left|\gamma_{1}-\gamma_{2}\right| \leqslant 1} \frac{\log \left(\left|\gamma_{1}\right|+3\right) \log \left(\left|\gamma_{2}\right|+3\right)}{\left|\gamma_{1} \gamma_{2}\right|} \int_{2^{n}}^{2^{n+1}} \iiint \int_{[0,2 \varepsilon]^{4}} e^{-y\left(v_{1}+v_{2}\right)} d u_{j} d v_{j} d y
\end{gathered}
$$

$\ll 2^{-n}$.

For the remaining sums, for brevity we define

$$
\rho_{1}=\frac{1}{2}+i \gamma_{1}, \quad \rho_{2}=\frac{1}{2}-i \gamma_{2} .
$$

Next,

$$
\begin{aligned}
\int_{2^{n}}^{2^{n+1}}\left|\Sigma_{2,3}(y)\right|^{2} d y=\int_{2^{n}}^{2^{n+1}} \sum_{\left|\gamma_{1}\right|,\left|\gamma_{2}\right| \leqslant T} m\left(\gamma_{1}\right) m\left(\gamma_{2}\right) e^{i y\left(\gamma_{1}-\gamma_{2}\right)} \rho_{1} \rho_{2} \\
\times \iiint \int_{\substack{[0,2 \varepsilon]^{4} \\
\left|u_{j}-v_{j}\right| \leqslant 2^{-n}}} \frac{\left(e^{-v_{1} y}-e^{-u_{1} y}\right)\left(e^{-v_{2} y}-e^{-u_{2} y}\right)}{\prod_{j=1}^{2}\left(u_{j}-v_{j}\right)\left(\rho_{j}-v_{j}\right)\left(\rho_{j}-u_{j}\right)} d v_{j} d v_{j} d y .
\end{aligned}
$$

By (5.1), the integrand in the quadruple integral is $\ll y^{2} e^{-u y-u_{1} y}\left|\rho_{1} \rho_{2}\right|^{-2}$. By Lemma2.2, for a given $\gamma_{1}$, there are $\ll \log \left(\left|\gamma_{1}\right|+3\right)$ zeros $\gamma_{2}$ with $\left|\gamma_{1}-\gamma_{2}\right|<1$. Hence, the contribution from terms with $\left|\gamma_{1}-\gamma_{2}\right|<1$ is

$$
\ll 2^{-n} \sum_{\left|\gamma_{1}-\gamma_{2}\right|<1} \frac{m\left(\gamma_{1}\right) m\left(\gamma_{2}\right)}{\left|\rho_{1} \rho_{2}\right|} \ll 2^{-n} \sum_{\gamma_{1}} \frac{\log ^{3}\left(\left|\gamma_{1}\right|+3\right)}{\left|\gamma_{1}\right|^{2}} \ll 2^{-n} .
$$

Using integration by parts, we have

$$
\int_{2^{n}}^{2^{n+1}} e^{i y\left(\gamma_{1}-\gamma_{2}\right)}\left(e^{-v_{1} y}-e^{-u_{2} y}\right)\left(e^{-v_{1} y}-e^{-u_{2} y}\right) d y \ll \frac{2^{3 n}\left|u_{1}-v_{1}\right|\left|u_{2}-v_{2}\right| e^{-2^{n}\left(u_{1}+u_{2}\right)}}{\left|\gamma_{1}-\gamma_{2}\right|}
$$

uniformly in $u_{1}, v_{1}, u_{2}, v_{2}$. Thus, by (5.2) and Lemma 2.4 the contribution from terms with $\left|\gamma_{1}-\gamma_{2}\right| \geqslant 1$ is

$$
\ll 2^{-n} \sum_{\left|\gamma_{1}-\gamma_{2}\right| \geqslant 1} \frac{m\left(\gamma_{1}\right) m\left(\gamma_{2}\right)}{\left|\rho_{1} \rho_{2}\right| \cdot\left|\gamma_{1}-\gamma_{2}\right|} \ll 2^{-n} .
$$

Combining these estimates, we have

$$
\int_{2^{n}}^{2^{n+1}}\left|\Sigma_{2,3}(y)\right|^{2} d y \ll 2^{-n}
$$

In the same manner, we have

$$
\int_{2^{n}}^{2^{n+1}}\left|\Sigma_{2,4}(y)\right|^{2} d y=\sum_{\substack{\left|\gamma_{1}\right| \leqslant T \\\left|\gamma_{2}\right| \leqslant T}} m\left(\gamma_{1}\right) m\left(\gamma_{2}\right) \rho_{1} \rho_{2} \int_{2^{n}}^{2^{n+1}} \iiint \int_{\substack{[0,2 \varepsilon]^{4} \\ u_{j} \leqslant v_{j}-2^{-n}}} \frac{e^{y\left(-v_{1}-v_{2}+i\left(\gamma_{1}-\gamma_{2}\right)\right)} d u_{j} d v_{j}}{\prod_{j=1}^{2}\left(u_{j}-v_{j}\right)\left(\rho_{j}-v_{j}\right)\left(\rho_{j}-u_{j}\right)} d y .
$$


The contribution to the right side from terms with $\left|\gamma_{1}-\gamma_{2}\right|<1$ is

$$
\begin{aligned}
& \ll \sum_{\left|\gamma_{1}-\gamma_{2}\right|<1} \frac{m\left(\gamma_{1}\right) m\left(\gamma_{2}\right)}{\left|\gamma_{1} \gamma_{2}\right|} \int_{2^{n}}^{2^{n+1}}\left(\int_{2^{-n}}^{2 \varepsilon} \int_{0}^{v-2^{-n}} \frac{e^{-y v}}{(v-u)} d u d v\right)^{2} \\
& \ll \sum_{\gamma_{1}} \frac{\log ^{3}\left(\left|\gamma_{1}\right|+3\right)}{\left|\gamma_{1}\right|^{2}} \int_{2^{n}}^{2^{n+1}}\left(\int_{1 / y}^{\infty} e^{-y v} \log (y v) d v\right)^{2} \ll 2^{-n} .
\end{aligned}
$$

The terms with $\left|\gamma_{1}-\gamma_{2}\right|>1$ contribute

$$
\begin{aligned}
& \ll \sum_{\substack{\left|\gamma_{1}\right|,\left|\gamma_{2}\right|<T \\
\left|\gamma_{1}-\gamma_{2}\right|>1}} \frac{m\left(\gamma_{1}\right) m\left(\gamma_{2}\right)}{\left|\gamma_{1} \gamma_{2}\right| \cdot\left|\gamma_{1}-\gamma_{2}\right|}\left(\int_{2^{-n}}^{2 \varepsilon} \int_{0}^{v-2^{-n}} \frac{e^{-2^{n} v}}{v-u} d u d v\right)^{2} \\
& \ll \sum_{\left|\gamma_{1}-\gamma_{2}\right|>1} \frac{\log \left(\left|\gamma_{1}\right|+3\right) \log \left(\left|\gamma_{2}\right|+3\right)}{\left|\gamma_{1} \gamma_{2}\right| \cdot\left|\gamma_{1}-\gamma_{2}\right|}\left(\frac{1}{2^{n}}\right)^{2} \ll \frac{1}{2^{2 n}} .
\end{aligned}
$$

Therefore,

$$
\int_{2^{n}}^{2^{n+1}}\left|\Sigma_{2,4}(y)\right|^{2} d y \ll 2^{-n} .
$$

Estimating an average of $\Sigma_{2,1}(y)$ is more complicated, since $R(\gamma, w)$ could be very large if $|w|$ is small and there is another $\gamma^{\prime}$ very close to $\gamma$. We get around the problem by noticing that $R(\gamma, w)+R(\gamma,-w)$ is always small. We first have, by (5.1) and Lemma 2.2.

$$
\begin{aligned}
\int_{2^{n}}^{2^{n+1}}\left|\Sigma_{2,1}(y)\right|^{2} d y \ll & \sum_{\gamma_{1}, \gamma_{2}} \log ^{2}\left(\left|\gamma_{1}\right|+3\right) \log ^{2}\left(\left|\gamma_{2}\right|+3\right) \max _{\substack{0<\left|\gamma_{1}-\gamma_{1}^{\prime}\right| \leqslant 1 \\
0<\left|\gamma_{2}-\gamma_{2}^{\prime}\right| \leqslant 1}} \int_{2^{n}}^{2^{n+1}} e^{i y\left(\gamma_{1}-\gamma_{2}\right)} \\
& \times \iiint \int_{[0,2 \varepsilon]^{4}} \frac{e^{y\left(-v_{1}-v_{2}\right)}}{\left(u_{1}-v_{1}+i \xi_{1}\right)\left(\rho_{1}-v_{1}\right)\left(u_{2}-v_{2}+i \xi_{2}\right)\left(\rho_{2}-v_{2}\right)} d u_{j} d v_{j} d y,
\end{aligned}
$$

where $\xi_{1}=\gamma_{1}-\gamma_{1}^{\prime}$ and $\xi_{2}=-\left(\gamma_{2}-\gamma_{2}^{\prime}\right)$. Let

$$
M(\gamma)=\max _{\substack{\left|\gamma-\gamma_{1}\right| \leqslant 1 \\ 0<\left|\gamma_{1}-\gamma_{1}^{\prime}\right|<1}} \frac{2}{\left|\gamma_{1}-\gamma_{1}^{\prime}\right|}
$$

By Lemmas 2.3 and 5.1, the terms with $\left|\gamma_{1}-\gamma_{2}\right|<1$ contribute

$$
\begin{aligned}
& \ll \sum_{\left|\gamma_{1}-\gamma_{2}\right|<1} \frac{\log ^{2}\left(\left|\gamma_{1}\right|+3\right) \log ^{2}\left(\left|\gamma_{2}\right|+3\right)}{\left|\gamma_{1} \gamma_{2}\right|} \int_{2^{n}}^{2^{n+1}} \frac{1}{y^{2}} \prod_{j=1}^{2} \log \left(\min \left(2 y, \frac{2}{\left|\gamma_{j}-\gamma_{j}^{\prime}\right|}\right)\right) d y \\
& \ll \frac{1}{2^{n}} \sum_{\gamma_{1}} \frac{\log ^{5}\left(\left|\gamma_{1}\right|+3\right)}{\left|\gamma_{1}\right|^{2}} \log ^{2}\left(\min \left(2^{n+2}, M(\gamma)\right)\right)=o\left(\frac{n^{2}}{2^{n}}\right) \quad(n \rightarrow \infty) .
\end{aligned}
$$

Now suppose $\left|\gamma_{1}-\gamma_{2}\right|>1$. With $\gamma_{1}, \gamma_{2}, \gamma_{1}^{\prime}, \gamma_{2}^{\prime}$ all fixed, let $\Delta=\gamma_{1}-\gamma_{2}$. Fixing $u_{1}, v_{1}, u_{2}, v_{2}$, we integrate over $y$ first. The quintuple integral in (5.6) is $J\left(2^{n+1}\right)-J\left(2^{n}\right)$, where

$$
J(y)=e^{i y \Delta} \iiint \int_{[0,2 \varepsilon]^{4}} \frac{e^{-y\left(v_{1}+v_{2}\right)}}{\left(i \Delta-v_{1}-v_{2}\right) \prod_{j=1}^{2}\left(u_{j}-v_{j}+i \xi_{j}\right)\left(\rho_{j}-v_{j}\right)} d u_{j} d v_{j} .
$$


Using

$$
\frac{1}{i \Delta-v_{1}-v_{2}}=\frac{1}{i \Delta} \sum_{k=0}^{\infty}\left(\frac{v_{1}+v_{2}}{i \Delta}\right)^{k}=\sum_{a, b \geqslant 0}\left(\begin{array}{c}
a+b \\
a
\end{array}\right) \frac{v_{1}^{a} v_{2}^{b}}{(i \Delta)^{a+b}},
$$

together with Lemma 5.1 yields

$$
|J(y)| \ll \frac{\log ^{2} y}{\left|\rho_{1} \rho_{2} \Delta\right| y^{2}} \sum_{a, b \geqslant 0}\left(\begin{array}{c}
a+b \\
a
\end{array}\right)\left(\frac{4 \varepsilon}{|\Delta|}\right)^{a+b} \ll \frac{\log ^{2} y}{\left|\rho_{1} \rho_{2} \Delta\right| y^{2}} .
$$

Therefore, by Lemma 2.4

$$
\sum_{\gamma_{1}, \gamma_{2}} \log ^{2}\left(\left|\gamma_{1}\right|+3\right) \log ^{2}\left(\left|\gamma_{2}\right|+3\right) \max _{\substack{0<\left|\gamma_{1}-\gamma_{1}^{\prime}\right| \leqslant 1 \\ 0<\left|\gamma_{2}-\gamma_{2}^{\prime}\right| \leqslant 1}}\left|J\left(2^{n+1}\right)-J\left(2^{n}\right)\right| \ll \frac{n^{2}}{2^{2 n}},
$$

and hence

$$
\int_{2^{n}}^{2^{n+1}}\left|\Sigma_{2,1}(y)\right|^{2}=o\left(n^{2} 2^{-n}\right)
$$

Define

$$
\Sigma_{2}(x ; \chi)=(\log x) \sum_{j=1}^{4} \Sigma_{2, j}(\log x)
$$

By (5.3), (5.4), (5.5) and (5.7),

$$
\int_{2}^{Y}\left|\Sigma_{2}\left(e^{y} ; \chi\right)\right|^{2} d y \leqslant 4 \sum_{j=1}^{4} \sum_{n \leqslant \frac{\log Y}{\log 2}+1} 2^{2 n} \int_{2^{n}}^{2^{n+1}}\left|\Sigma_{2, j}(y)\right|^{2} d y=o\left(Y \log ^{2} Y\right) \quad(Y \rightarrow \infty) .
$$

This completes the proof of Theorem 3.2

\section{Proof of Lemma 3.3}

Put $y=\log x$. For any $\gamma$ we have

$$
\begin{aligned}
\int_{0}^{2 \varepsilon-2^{-n}} & \frac{e^{-y v}}{\frac{1}{2}-v+i \gamma} \int_{v+2^{-n}}^{2 \varepsilon} \frac{d u}{(u-v)\left(\frac{1}{2}-u+i \gamma\right)} d v \\
& =\int_{0}^{2 \varepsilon-2^{-n}} e^{-y v}\left(\frac{1}{\frac{1}{2}+i \gamma}+O\left(\frac{v}{\frac{1}{4}+\gamma^{2}}\right)\right) \int_{v+2^{-n}}^{2 \varepsilon}\left(\frac{1}{\frac{1}{2}+i \gamma}+O\left(\frac{u}{\frac{1}{4}+\gamma^{2}}\right)\right) \frac{d u}{u-v} d v \\
& =\frac{M+E}{(1 / 2+i \gamma)^{2}}
\end{aligned}
$$

where

$$
M=\int_{0}^{2 \varepsilon-2^{-n}} e^{-y v}\left(\log (2 \varepsilon-v)+\log 2^{n}\right) d v=\frac{\log y+O(1)}{y}
$$

and

$$
\begin{aligned}
E & \ll \int_{0}^{2 \varepsilon-2^{-n}} e^{-y v} \int_{v+2^{-n}}^{2 \varepsilon} \frac{u}{u-v} d u d v \\
& \ll \int_{0}^{2 \varepsilon-2^{-n}} e^{-y v}\left(1+v \log 2^{n}+v \log (2 \varepsilon-v)\right) d v \ll \frac{1}{y} .
\end{aligned}
$$


Hence, the zeros with $|\gamma| \leqslant T_{0}$ contribute

$$
\frac{2 \log \log x}{\log x} \sum_{\substack{|\gamma| \leqslant T_{0} \\ \gamma \text { distinct }}} \frac{m^{2}(\gamma) x^{i \gamma}}{1 / 2+i \gamma}+O\left(\frac{\log ^{3} T_{0}}{\log x}\right) .
$$

Next, let $\Sigma_{3}\left(x ; T_{0}\right)$ be the sum over zeros with $T_{0}<|\gamma| \leqslant T$. We have

$$
\begin{gathered}
\int_{2^{n}}^{2^{n+1}}\left|\Sigma_{3}\left(e^{y}, T_{0}\right)\right|^{2} d y \leqslant \sum_{T_{0} \leqslant\left|\gamma_{1}\right|,\left|\gamma_{2}\right| \leqslant T} 2^{2 n+2} m\left(\gamma_{1}\right) m\left(\gamma_{2}\right)\left(\frac{1}{2}+i \gamma_{1}\right)\left(\frac{1}{2}-i \gamma_{2}\right) \\
\int_{2^{n}}^{2^{n+1}} e^{y i\left(\gamma_{1}-\gamma_{2}\right)} \iiint \int_{u_{j} \geqslant v_{j}+2^{-n}} \frac{e^{-y v_{1}-y v_{2}}}{\prod_{j=1}^{2}\left(u_{j}-v_{j}\right)\left(\frac{1}{2}-v_{j}+i \gamma_{j}\right)\left(\frac{1}{2}-u_{j}+i \gamma_{j}\right)} d u_{j} d v_{j} d y .
\end{gathered}
$$

The sum over $\left|\gamma_{1}-\gamma_{2}\right|<1$ on the right side of (6.1) is

$$
\begin{aligned}
& \ll \sum_{\substack{T_{0} \leqslant\left|\gamma_{1}\right|,\left|\gamma_{2}\right| \leqslant T \\
\left|\gamma_{1}-\gamma_{2}\right|<1}} \frac{2^{2 n} m\left(\gamma_{1}\right) m\left(\gamma_{2}\right)}{\left|\gamma_{1}\right|\left|\gamma_{2}\right|} \int_{2^{n}}^{2^{n+1}} \iiint \int_{u_{j} \geqslant v_{j}+2^{-n}} \frac{e^{-y v_{1}-y v_{2}}}{\left(u_{1}-v_{1}\right)\left(u_{2}-v_{2}\right)} d u_{j} d v_{j} d y \\
& \ll \sum_{\substack{T_{0} \leqslant\left|\gamma_{1}\right|,\left|\gamma_{2}\right| \leqslant T \\
\left|\gamma_{1}-\gamma_{2}\right|<1}} \frac{n^{2} 2^{n} m\left(\gamma_{1}\right) m\left(\gamma_{2}\right)}{\left|\gamma_{1}\right|\left|\gamma_{2}\right|} \ll n^{2} 2^{n} \sum_{|\gamma| \geqslant T_{0}} \frac{\log ^{3}(|\gamma|+3)}{|\gamma|} \ll \frac{n^{2} 2^{n} \log ^{5} T_{0}}{T_{0}},
\end{aligned}
$$

applying Lemma2.2. The terms where $\left|\gamma_{1}-\gamma_{2}\right|>1$ on the right hand side of (6.1) total

$$
\begin{aligned}
& \ll \sum_{\substack{T_{0} \leqslant\left|\gamma_{1}\right|,\left|\gamma_{2}\right| \leqslant T \\
\left|\gamma_{1}-\gamma_{2}\right|>1}} \frac{2^{2 n} m\left(\gamma_{1}\right) m\left(\gamma_{2}\right)}{\left|\gamma_{1}\right|\left|\gamma_{2}\right|\left|\gamma_{1}-\gamma_{2}\right|} \iiint \int_{u_{j} \geqslant v_{j}+2^{-n}} \frac{e^{-2^{n} v_{1}-2^{n} v_{2}}}{\left(u_{1}-v_{1}\right)\left(u_{2}-v_{2}\right)} d u_{j} d v_{j} \\
& \ll \sum_{\substack{T_{0} \leqslant\left|\gamma_{1}\right|,\left|\gamma_{2}\right| \\
\left|\gamma_{1}-\gamma_{2}\right|>1}} \frac{n^{2} \log \left(\left|\gamma_{1}\right|+3\right) \log \left(\left|\gamma_{2}\right|+3\right)}{\left|\gamma_{1}\right|\left|\gamma_{2}\right|\left|\gamma_{1}-\gamma_{2}\right|} \ll n^{2} \frac{\log ^{5} T_{0}}{T_{0}} .
\end{aligned}
$$

by Lemma2.4 Summing over $n$ proves the lemma.

\section{REFERENCES}

[Ch] P. L. Chebyshev, Lettre de M. le professeur Tchébyshev á M. Fuss, sur un nouveau théoreme rélatif aux nombres premiers contenus dans la formes $4 n+1$ et $4 n+3$, Bull. de la Classe phys.-math. de l'Acad. Imp. des Sciences St. Petersburg 11 (1853), 208.

[Da] H. Davenport, Multiplicative Number Theory, 3rd ed., Graduate Texts in Mathematics vol. 74, Springer-Verlag, New YorkBerlin, 2000.

[FM] D. Fiorilli and G. Martin, Inequities in the Shanks-Rényi Prime Number Race: An asymptotic formula for the densities , (pre-print, 2009, arXiv:0912.4908).

[FK] K. Ford and S. Konyagin, Chebyshev's conjecture and the prime number race. IV International Conference "Modern Problems of Number Theory and its Applications": Current Problems, Part II (Russian) (Tula, 2001), 67-91, Mosk. Gos. Univ. im. Lomonosova, Mekh.-Mat. Fak., Moscow, 2002.

[GM] A. Granville and G. Martin, Prime number races. Amer. Math. Monthly 113 (2006), no. 1, 1-33.

[KT] S. Knapowski and P. Turán, Comparative Prime Number Theory I., Acta. Math. Sci. Hungar. 13 (1962), $315-342$.

[La] E. Landau, Handbuch der Lehre von der Verteilung der Primzahlen, 3rd ed., Chelsea, New York, 1974.

[Le] J. Leech, Note on the distribution of prime numbers, J. London Math. Soc. 32 (1957), 56-58.

[Li] J. E. Littlewood, Sur la distribution des nombres premiers, C. R. Acad. des Sciences Paris 158 (1914), 1869-1872.

[RS] M. Rubinstein and P. Sarnak, Chebyshev's Bias, J. Exper. Math. 3 (1994), 173-197.

[S] J. Sneed, Lead changes in the prime number race, Math. Comp. (to appear). 
E-mail address: ford@math.uiuc.edu, jpsneed@uiuc.edu

Department of Mathematics, University of Illinois at Urbana-Champaign, 1409 West Green St., UrBANA, IL 61801 